\title{
Effects of a dietary strawberry powder on parameters of vascular health in adolescent males
}

\author{
Dragana Djurica ${ }^{1}$, Roberta R. Holt ${ }^{1}$, Jingyan Ren ${ }^{1}$, Alan W. Shindel ${ }^{2}$, Robert M. Hackman ${ }^{1}$ and \\ Carl L. Keen ${ }^{1,3 *}$ \\ ${ }^{1}$ Department of Nutrition, University of California, Davis, One Shields Avenue, Davis, CA 95616, USA \\ ${ }^{2}$ Department of Urology, University of California Davis Medical Center; 4860 Y Street, Suite 2200 Sacramento, CA 95817, USA \\ ${ }^{3}$ Department of Internal Medicine, University of California Davis Medical Center; 4150 V Street, Suite 3100 Sacramento, \\ CA 95817, USA \\ (Submitted 9 November 2015 - Final revision received 21 April 2016 - Accepted 17 May 2016 - First published online 20 June 2016)
}

\begin{abstract}
Previous studies have shown that the intake of freeze-dried strawberry powder (FDSP) improves select markers of cardiovascular health in adults with cardiovascular risk factors; however, whether these improvements can be observed in at-risk adolescents is unknown. A randomised, double-blind, cross-over study enrolled twenty-five overweight or obese males, aged 14-18 years, to consume $50 \mathrm{~g}$ of a FDSP or a control powder, daily for 1 week. Before and after each test period, measures of microvascular function, plasma nitrate/nitrite, platelet reactivity and blood lipids were collected at baseline and acutely $1 \mathrm{~h}$ after FDSP intake. Acute plasma nitrate/nitrite levels increased $1 \mathrm{~h}$ after consuming the FDSP during Study Visit 1 before daily FDSP intake $(P<0 \cdot 001)$ and during Study Visit 2 after 1 week of FDSP intake $(P<0 \cdot 001)$ compared with control powder intake. As a group, fasting nitrate/nitrite levels did not significantly change after 1 week of control or FDSP intake. However, for those individuals where fasting nitrate levels increased after short-term FDSP intake compared with controls, an increase in reactive hyperaemia index (RHI) was observed $(P=0.014)$, whereas RHI was unchanged in those individuals who did not have a significant increase in nitrate $(P=0.396)$. Taken together, these data support the concept that strawberries can provide vascular health benefits to heavier adolescent males.
\end{abstract}

Key words: Peripheral arterial tonometry: Strawberry powder: Obese adolescents: Nitrate

Obesity is a major public health issue in both adult and paediatric populations ${ }^{(1)}$, particularly as a risk factor for the development of CVD, a leading cause of morbidity and mortality in many countries ${ }^{(2)}$. Increasing adiposity contributes to the development of atherosclerotic CVD, in part through the promotion of a chronic, low-grade inflammatory state and through endothelial dysfunction ${ }^{(3,4)}$, which is typically characterised by reduced nitric oxide (NO) bioavailability ${ }^{(4,5)}$. $\mathrm{NO}$ is anti-atherogenic, acting as a key endothelium-derived vasodilator that reduces cellular proliferation, inflammation and platelet activation $^{(6)}$. Although clinical manifestations of atherosclerosis are typically not observed in adolescents, CVD has a long asymptomatic phase of development, which can begin early in life ${ }^{(7)}$. Moreover, the changes in vasculature that are associated with an increased fat mass in older individuals can be observed in overweight and obese paediatric populations ${ }^{(8-10)}$. As an increased BMI in children and adolescents is associated with increased cardiovascular (CV) risk burden ${ }^{(8)}$, identification of children who are at risk for atherosclerosis and the introduction of early lifestyle and dietary interventions that reduce this risk are important public as well as personal health goals.

Diets rich in fruits and vegetables can promote health and attenuate, or delay, the onset of various chronic diseases including $\mathrm{CVD}^{(11,12)}$. Besides being a good source of several essential nutrients including numerous vitamins, minerals, fatty acids and fibre, many plant foods are rich in polyphenols - a diverse family of compounds that can provide certain CV benefits ${ }^{(13)}$. Strawberries are rich in certain polyphenols, which may help explain why the intake of freeze-dried strawberry powder (FDSP) has been reported to improve lipid profiles, insulin sensitivity and attenuate inflammatory and thrombotic responses in adult populations with or without the metabolic syndrome ${ }^{(14-17)}$. In the present study, we evaluated the effects of short-term FDSP intake in adolescent males who were at or above the 75th percentile for weight on microvascular function as measured by peripheral arterial tonometry (PAT), as well as effects on platelet reactivity and plasma nitrate/nitrite concentrations.

Abbreviations: CV, cardiovascular; FDSP, freeze-dried strawberry powder; fRHI, Framingham reactive hyperaemia index; NO, nitric oxide; PAT, peripheral arterial tonometry; RHI, reactive hyperaemia index.

* Corresponding author: C. L. Keen, fax +1 530752 8966, email clkeen@ucdavis.edu 


\section{Methods}

\section{Participants}

Healthy, male adolescents (14-18 years of age) from the Sacramento, California, metropolitan region were recruited via flyers and newspaper advertisements. Inclusion criteria included a BMI for age and sex at or above the 75th percentile (BMI percentile), based on data from the United States Center for Disease Control, and a willingness to comply with the study protocols. Exclusion criteria included use of medications that affect vascular function, use of dietary supplements, chronic/ routine high-intensity exercise or participation in organised sports, and an inability to wear PAT probes and/or to remain still and quiet during test procedures. Participants were instructed to refrain from consuming berries throughout the study period. All analyses were conducted in accordance with the Declaration of Helsinki. The Institutional Review Board of the University of California, Davis, approved the study protocol, and all participants and their parents or their legal guardians were asked to provide written informed assent and/or consent before enrolment.

\section{Study design}

A randomised, controlled, double-blind, cross-over study was conducted that examined both the short-term (1 week) and acute $(1 \mathrm{~h})$ vascular responses to strawberry intake. Participants were randomised by block design to consume $50 \mathrm{~g}$ of FDSP or a control powder for 1 week, and after a 1 week washout period they were instructed to consume the alternate test powder for an additional week. The treatment and washout periods, as well as the 1-h acute sampling point, were chosen on the basis of previous reports of improved vascular function after the intake of flavonoid-rich foods ${ }^{(18,19)}$, with peak plasma levels of strawberry metabolites obtained approximately $1 \mathrm{~h}$ after intake $^{(20)}$. Randomisation was performed following a plan formulated via a web-based random number generator (www. randomization.com). The isoenergetically matched powders were produced and provided by the California Strawberry Commission (Table 1), with a daily intake of $50 \mathrm{~g}$ of FDSP estimated to provide $500 \mathrm{~g}$ in fresh weight of strawberries (equivalent to three servings) ${ }^{(14)}$. The control powder was matched for energy content and sugars, was similar in colour and flavour to the test powder, but provided no additional protein or flavonoids, and was significantly reduced in its $\mathrm{K}$ and fibre contents. Both powders were pre-packaged in $25 \mathrm{~g}$ servings, and mixed with water to produce a shake beverage for consumption. All participants were asked to return any used or unused beverage packages as an assessment of compliance.

Study day assessments were conducted before commencement of daily powder intake (Study Visit 1) and at the end of each 1-week period of daily powder intake (Study Visit 2). For each study visit, the participants were asked to arrive in the morning after an overnight fast. After weight and blood pressure were measured, a baseline measurement of microvascular function, assessed by PAT, was carried out, and blood samples were collected. Immediately afterwards, the study participants consumed $50 \mathrm{~g}$ of their assigned test beverage; $1 \mathrm{~h}$
Table 1. Powder composition

\begin{tabular}{lcc}
\hline & Control powder & FDSP \\
\hline Energy content & 180 & 172 \\
Total carbohydrates $(\mathrm{g} / 50 \mathrm{~g})$ & 42 & 39 \\
Total sugars $(\mathrm{g} / 50 \mathrm{~g})$ & 31 & 28 \\
Glucose $(\mathrm{g} / 50 \mathrm{~g})$ & 9 & 10 \\
Fructose $(\mathrm{g} / 50 \mathrm{~g})$ & 12 & 11 \\
Sucrose $(\mathrm{g} / 50 \mathrm{~g})$ & 10 & 7 \\
Total dietary fibre $(\mathrm{g} / 50 \mathrm{~g})$ & 4 & 8 \\
Protein $(\mathrm{g} / 50 \mathrm{~g})$ & 0 & 3 \\
K (mg/50 g) & 350 & 839 \\
Phenolics (mg/50 g) & & \\
Gallic acid & 0 & 0.20 \\
3,4-Dihydrobenzoic acid & 0 & 0.08 \\
Procyanidin B1 & 0 & 15.31 \\
(+)-Catechin & 0 & 12.52 \\
Cyanidin-3-glucoside & 0 & $5 \cdot 82$ \\
Syringic acid & 0 & 0.01 \\
Pelargonidin-3-glucoside & 0 & 198.5 \\
p-Coumaric acid & 0 & $0 \cdot 13$ \\
2-Hydroxycinnamic acid & 0 & $0 \cdot 10$ \\
Rutin & 0 & 1.68 \\
Ellagic acid & 0 & 6.3 \\
Isoquercetin & 0 & 3.31 \\
Sinapic acid & 0 & $0 \cdot 20$ \\
Tiliroside & 0.37 \\
Quercetin & 0 & $0 \cdot 73$ \\
Kaempferol & $0 \cdot 18$ \\
\hline
\end{tabular}

FDSP, freeze-dried strawberry powder.

following this intake, a second PAT measurement was carried out and blood samples were collected, which completed the study visit. Participants were then provided with packets of powder for the 1-week intervention period, and were instructed to consume one packet at breakfast and a second at dinner. Apart from being instructed to refrain from consuming berries throughout the study period, participants were instructed to continue their normal diet and to complete a 3 -d food record during each treatment period, which included 2 weekdays and 1 weekend day. Food records were analysed using The Food Processor SQL (version 10.1.0; ESHA Research).

\section{Microvascular function}

Microvascular function was assessed by PAT (Endo-PAT2000; Itamar Medical) as previously described ${ }^{(21)}$. In brief, all participants acclimated to a quiet and temperature-controlled room by resting in the supine position for $30 \mathrm{~min}$. Following supine blood pressure measurement, the PAT measurement was performed in the supine position, which involved a 5-10 min baseline recording, followed by a 5 -min occlusion period, which was induced by inflating a blood pressure cuff placed on the proximal forearm to approximately $60 \mathrm{mmHg}$ above systolic blood pressure. After $5 \mathrm{~min}$, the pressure was released, and the resulting reactive hyperaemia response was recorded for an additional 3-5 min. The system software automatically calculated the reactive hyperaemia index (RHI), which is the ratio of the average of pulse wave amplitude (PWA) during a 1-min period following $90 \mathrm{~s}$ of reactive hyperaemia to the average PWA during a 3.5-min baseline period. In additional, the Framingham reactive hyperaemia 
index (fRHI) was calculated, which used the natural logarithmic transformation of the RHI ratio during data collection from 90 to $120 \mathrm{~s}$ after release of the occlusion. The fRHI has been reported to be strongly correlated with $\mathrm{CV}$ risk factors ${ }^{(22)}$.

\section{Plasma nitrate/nitrite}

Plasma nitrate and nitrite (nitrate/nitrite) were assessed using a nitrate/nitrite colorimetric assay (Cayman Chemical Co.). EDTA-treated plasma was separated from the whole blood by centrifugation $\left(15 \mathrm{~min}\right.$ at $1800 \mathrm{~g}$ and $4^{\circ} \mathrm{C}$ ) immediately after collection. The separated plasma was stored at $-80^{\circ} \mathrm{C}$ until analysis, processed according to the manufacturer's protocol and assessed in triplicate.

\section{Assessment of platelet activation markers}

The surface expression of platelet activation markers such as P-selectin and the active conformation of glycoprotein IIb/IIIa were assessed as previously described ${ }^{(23)}$. In brief, $50 \mu \mathrm{l}$ of citrated $(3.2 \%$ sodium citrate) whole blood was activated for 5 min with ADP or epinephrine at a final concentration of $20 \mu \mathrm{mol} / 1$ each. Subsequently, the activated blood was diluted with saline, and a 100- $\mu$ l aliquot was added to a second set of tubes containing the following antibodies: PAC1-FITC (clone PAC-1; BD Biosciences), CD62P-APC (clone AK-4; BD Biosciences) and CD42a-PE (clone ALMA.16; BD Biosciences). After $20 \mathrm{~min}$ of incubation, the samples were fixed with $1 \%$ paraformaldehyde; 10000 CD42a-positive events were collected on a flow cytometer (LSR II; Becton Dickinson). Activated platelets were defined as the percentage of CD42a events expressing PAC-1 (GPIIb/IIIa activated conformation) or CD62P (P-selectin)

\section{Additional blood measurements}

Blood samples were analysed for a comprehensive metabolic panel, lipid panel and complete blood count by the University of California Davis Medical Center Pathology Department.

\section{Data analysis}

Normally distributed data are expressed as means with their standard errors or standard deviations, as noted. Transformed data are expressed as back-transformed means and 95\% confidence intervals. Data were initially assessed for normality and absence of outliers, and those not normally distributed were determined by the Shapiro-Wilk test, were log-transformed and re-checked for normality. Statistical analyses of the normally distributed data were conducted using the repeated-measures ANOVA (rANOVA) with Bonferroni post hoc CI. Main effects and their interactions were assessed with rANOVA for Treatment (control and FDSP) and Visit ( $0 \mathrm{~h}$ both before, and after 1 week of intake) for short-term intake as well as for the acute

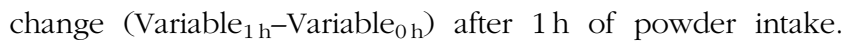
Interactive effects for period of intake (Control $\rightarrow$ FDSP or FDSP $\rightarrow$ Control) were also assessed for Visit 1 of each treatment group using ANOVA with period as a factor. Data that were not normally distributed were analysed via nonparametric Wilcoxon's signed-rank tests or Friedman's two-way
ANOVA by ranks test, with post boc analysis with Wilcoxon's signed-rank test with a Bonferroni correction. $P$ values of 0.05 or less were considered statistically different. Platelet data are presented as medians and interquartile ranges with all other data presented as means and standard deviations or means and 95\% confidence intervals. Analyses were performed with IBM SPSS software (version 22.0.0.0). Complete data sets were not available for select outcomes because of equipment or analysis failure. Therefore, the final subject total for PAT and nitrate/nitrite analysis was 24 for total cholesterol (TC), HDL and cholesterol:HDL was 25 and for LDL it was 22.

\section{Results}

\section{Population characteristics}

In all, twenty-eight adolescent males were enrolled between August 2013 and June 2014, of which twenty-five completed the study (Fig. 1). The initial treatment assignment was fifteen for FDSP intake and ten for control powder intake. The mean age of the study population was 16 years. On average, the participants were overweight with normal blood pressure, fasting lipids and blood glucose levels (Table 2). No main or interaction effects for BMI percentile were noted, but a significant Treatment $\times$ Visit interaction was observed for BMI $z$-score $\left(F_{1,23}=9.91, P=0 \cdot 005\right.$; Table 2$)$. The majority of participants did not return their used and unused treatment. Complete 3-d food records were obtained from seventeen of the subjects. Reported baseline energy and macronutrient intakes were similar between the two groups and did not differ at the end of the study, except for a decrease in the percentage of total $\mathrm{kJ}$ (kcal) from fat in the FDSP group (Table 2).

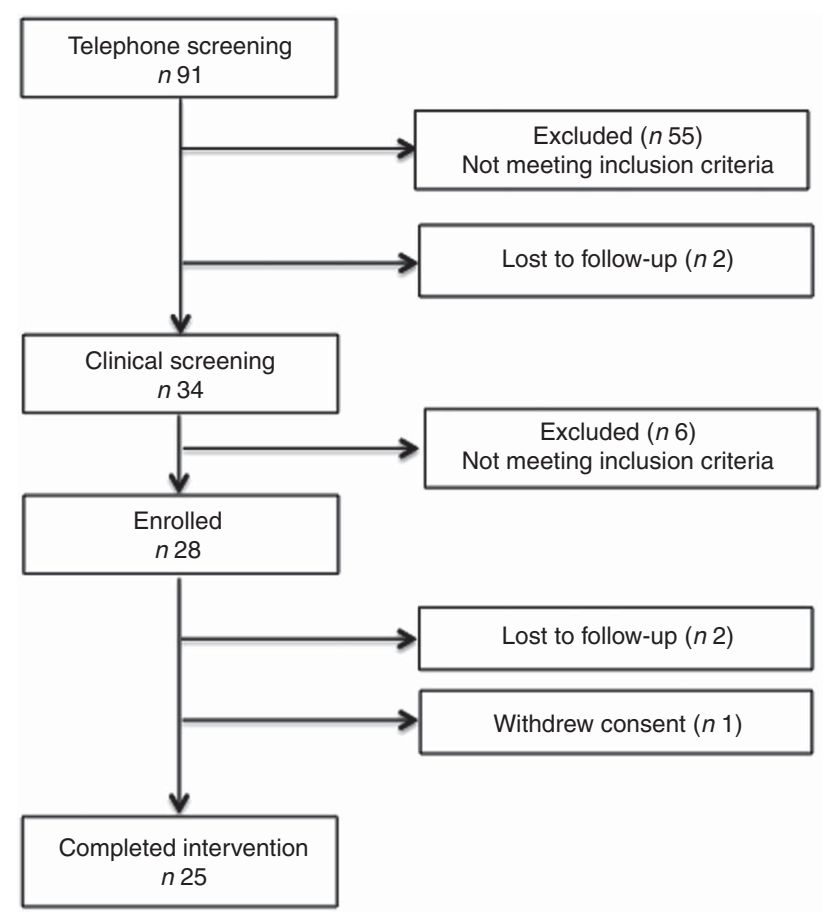

Fig. 1. Participant flow chart. 
Table 2. Estimated nutrient intake (Mean values and standard deviations)

\begin{tabular}{|c|c|c|c|c|c|}
\hline \multirow[b]{2}{*}{ Nutrients } & \multicolumn{2}{|c|}{ Control powder } & \multicolumn{2}{|c|}{ FDSP } & \multirow[b]{2}{*}{$P$} \\
\hline & Mean & SD & Mean & SD & \\
\hline Energy (kJ) & $7453 \cdot 0$ & 2676.5 & $7585 \cdot 6$ & $3087 \cdot 8$ & 0.824 \\
\hline Energy (kcal) & $1781 \cdot 3$ & 639.7 & 1813.0 & $738 \cdot 0$ & 0.824 \\
\hline Protein (g) & $68 \cdot 7$ & $25 \cdot 6$ & $67 \cdot 2$ & 30.4 & 0.812 \\
\hline Carbohydrates (g) & 224.7 & 84.5 & $248 \cdot 1$ & 102.5 & 0.249 \\
\hline Fat (\% energy) & $35 \cdot 0$ & 5.5 & 30.9 & 4.9 & $0.026^{*}$ \\
\hline Fat $(g)$ & $70 \cdot 0$ & 28.5 & 63.0 & 28.5 & 0.257 \\
\hline SFA (\% energy) & $12 \cdot 0$ & 3.0 & $10 \cdot 9$ & $2 \cdot 4$ & 0.130 \\
\hline SFA (g) & $23 \cdot 6$ & 9.6 & $22 \cdot 3$ & $10 \cdot 9$ & 0.547 \\
\hline MUFA (\% energy) & $6 \cdot 24$ & $4 \cdot 0$ & 6.56 & $2 \cdot 9$ & 0.721 \\
\hline MUFA (g) $\dagger$ & $13 \cdot 1$ & $12 \cdot 3$ & $13 \cdot 9$ & $8 \cdot 3$ & 0.246 \\
\hline PUFA (\% energy) & 2.99 & $1 \cdot 1$ & 3.23 & $1 \cdot 6$ & 0.655 \\
\hline PUFA $(g) \dagger$ & 5.98 & 3.3 & 6.87 & $5 \cdot 0$ & 0.619 \\
\hline Fibre $(\mathrm{g}) \dagger$ & 14.9 & $6 \cdot 4$ & $13 \cdot 7$ & $7 \cdot 1$ & 0.619 \\
\hline $\mathrm{Ca}(\mathrm{mg}) \dagger$ & 683.4 & $342 \cdot 9$ & $815 \cdot 2$ & 513.1 & 0.309 \\
\hline $\mathrm{K}(\mathrm{mg})$ & $1239 \cdot 4$ & 653.2 & 1156.5 & $497 \cdot 1$ & 0.617 \\
\hline $\mathrm{Na}(\mathrm{mg}) \dagger$ & 3073.4 & $1565 \cdot 4$ & $2988 \cdot 7$ & $1118 \cdot 2$ & 0.795 \\
\hline Caffeine $(\mathrm{mg}) \dagger$ & $72 \cdot 1$ & 264.1 & $32 \cdot 6$ & 56.4 & 0.182 \\
\hline
\end{tabular}

FDSP, freeze-dried strawberry powder

* Significantly different compared with the control group $(P<0.05)$.Statistical analysis by paired $t$ test or +Wilcoxon's signed-rank test.

\section{Microvascular function}

No acute or short-term effects of daily powder intake on blood pressure were observed (Table 3). Likewise, no statistically significant main or interaction effects for either short-term or acute change in RHI or fRHI were noted.

\section{Platelet activation markers}

The expressions of platelet activation markers were not significantly different at baseline before either FDSP or control powder intake (Table 4). Significant reductions in ADP-induced P-selectin expression were detected with short-term powder intake $(P<0.0001 ; n$ 24), with a decrease in ADP-induced P-selectin expression after 1 week of powder intake for both control powder $(30 \cdot 0(22 \cdot 2,43.7) \%$ on the first day of the study v. $27 \cdot 6(17 \cdot 3,40 \cdot 5) \%$ after 1 week of intake; $P=0 \cdot 01)$ as well as after FDSP intake $(32.4(21 \cdot 2,41 \cdot 4) \%$ on day $1 v .26 \cdot 2(18 \cdot 3$, $39 \cdot 3) \%$ after 1 week; $P=0 \cdot 02)$. No other significant changes in platelet activation were observed with either single serving or acute intake (Table 4).

\section{Plasma lipids}

No interactive effects were observed in plasma lipids (Table 3). Significant main effects for Visit were noted for LDL, HDL and cholesterol:HDL, with an overall decrease in LDL (mean difference $-0.2(95 \% \mathrm{CI}-0.3,-0.08) \mathrm{mmol} / \mathrm{l}, P=0.002)$ and cholesterol:HDL $(-0.005$ (95\% CI $-0.01,-0.003) \mathrm{mmol} / \mathrm{l}, \quad P=0.006)$, and an increase in HDL (0.0005 (95\% CI 0.00, 0.008) $\mathrm{mmol} / \mathrm{l}, P=0.048)$ was observed during Visit 2 compared with Visit 1, which was not significantly influenced by the period of intake. In addition, significant main effects for Treatment were observed for the acute 1-h change in cholesterol and non-HDL-cholesterol, with both measures increasing in the FDSP group compared with the control

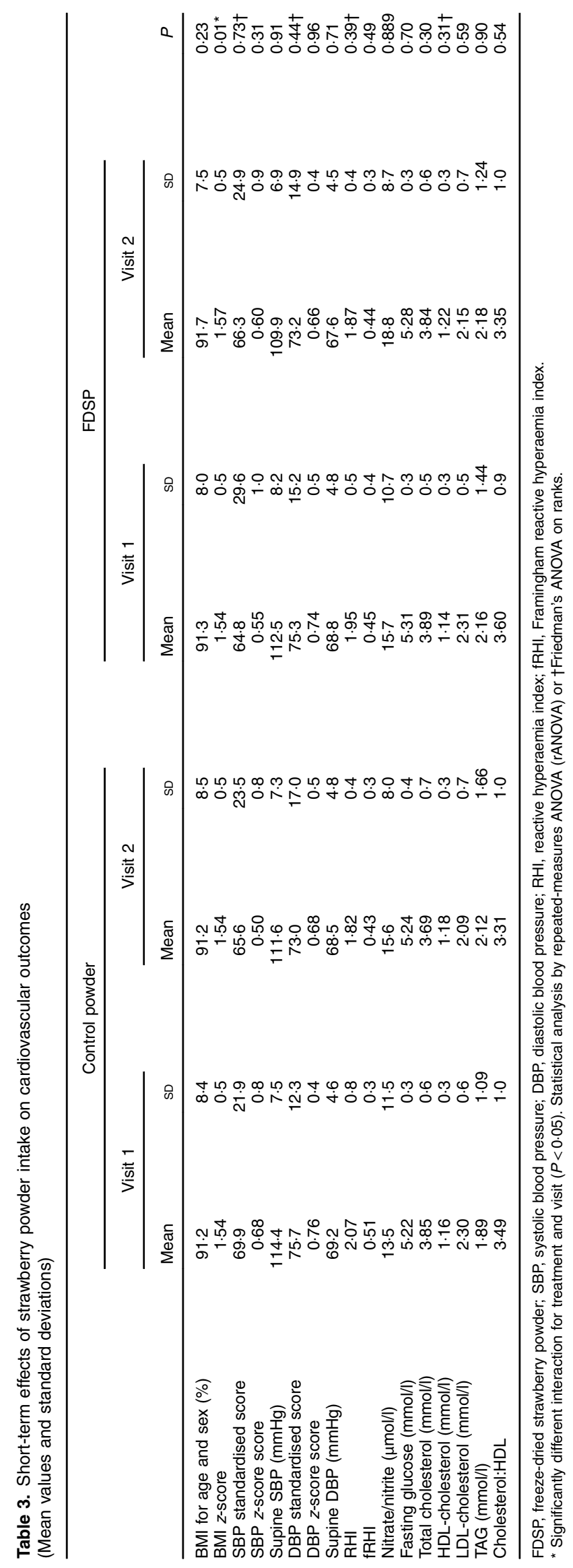


Strawberry powder intake in adolescent males

group (0.005 (95\% CI 0.0003, 0.001) $\mathrm{mmol} / 1, P=0.007$; and 0.0008 (95\% CI 0.0003, 0.001) mmol/l, $P=0.02$; respectively).

\section{Plasma nitrate/nitrite concentration}

Significant main effects for Treatment and Visit were observed for plasma concentration of nitrate/nitrite after 1 week of intake $\left(F_{1,23}=5.57, P=0.027\right.$ and $F_{1,23}=9.54, P=0.005$, respectively), with significantly greater increase in the FDSP group than in the control group (mean difference 0.89 (95\% CI 0.01, 0.17) and Visit $2 v$. Visit $1(0 \cdot 11(95 \%$ CI $0.04,0 \cdot 19))$. Interactions for Visit $\times$ Treatment did not reach statistical significance (Table 3, $P=0.889)$. A significant increase in the 1 -h acute change in nitrate/ nitrite levels $\left(\chi^{2}=58.3, P<0 \cdot 001\right)$ was observed after the intake of FDSP compared with that of the control powder during V1 (16.2 (SD 5.58) $v .-1.27$ (SD 2.51) $\mu \mathrm{mol} / 1$, respectively; $Z=-2.04$, $P<0.001$ ) and during V2 (-1.63 (sD 2.27) $v .15 .9$ (sD 6.01) $\mu \mathrm{mol} / \mathrm{l}$, respectively; $Z=-1.96, P<0.001)$. The magnitude of these effects is depicted as the change in plasma nitrate/nitrite (i.e. plasma nitrate/nitrite 1h $_{\text {- plasma nitrate/nitrite }}$ baseline before intake) in Fig. 2.

As the above data demonstrate that FDSP intake can induce a significant increase in plasma nitrate/nitrite levels in some individuals, the relationship between the change in plasma nitrate/nitrite levels and microvascular response was further explored. As described above, no significant interaction effect was noted in general for fasting plasma nitrate levels (i.e. Treatment $\times$ Visit), which can also be expressed as the shortterm change in plasma nitrate/nitrite between the two groups

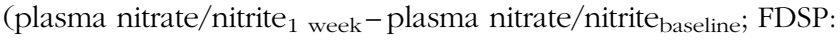
3.31 (sD 7.32) $\mu \mathrm{mol} / \mathrm{l} v$. Control: 1.76 (sD 9.28) $\mu \mathrm{mol} / \mathrm{l} ; Z=-0 \cdot 44$, $P=0 \cdot 6612, n$ 22). However, when these data were subdivided into those who had a positive 1-week change in fasting plasma nitrate/nitrite levels with FDSP intake compared with the control powder (responders) and those who had a negative change in plasma levels with FDSP intake compared with

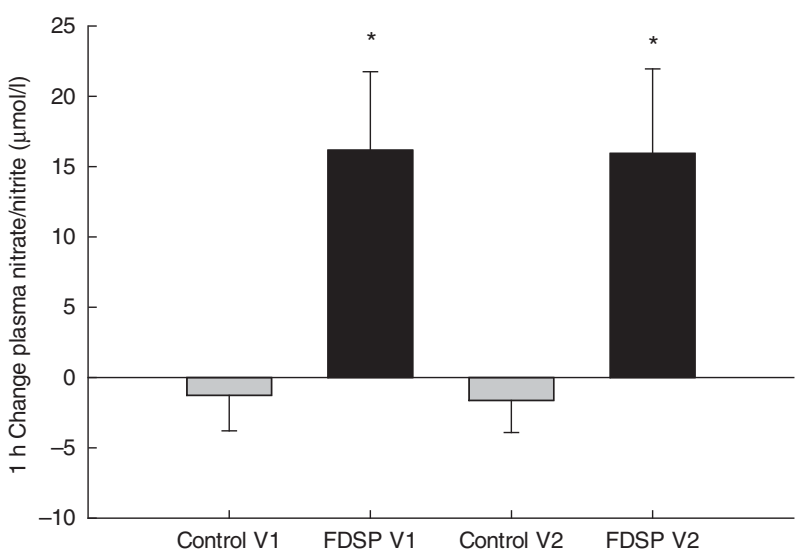

Fig. 2. Significant changes in plasma nitrate/nitrite expressed as the acute change in plasma nitrate/nitrite from baseline. Although the $1 \mathrm{~h}$ change in plasma nitrate/nitrite levels was not significant between study visits for either powder (control powder: $P=0.655$; freeze-dried strawberry powder (FDSP): $P=0.502$ ), FDSP intake did significantly increase plasma nitrate and nitrite concentrations during both study visits, compared with control powder intake ( $Z=-2.04$ and $Z=-1.96, P<0.001$ for both Study Visits 1 (V1) and 2 (V2), respectively). *Significantly different compared with the control group $(P<0.05)$. Friedman's two-way ANOVA on ranks. 
(a)

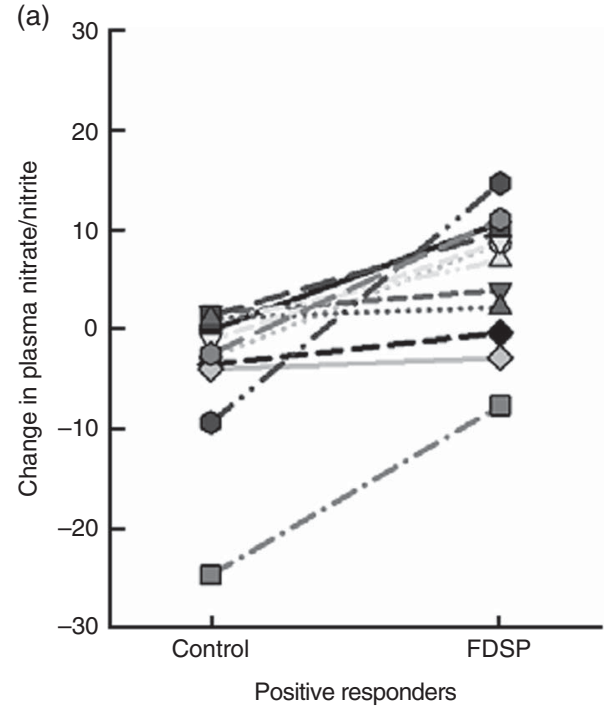

(c)

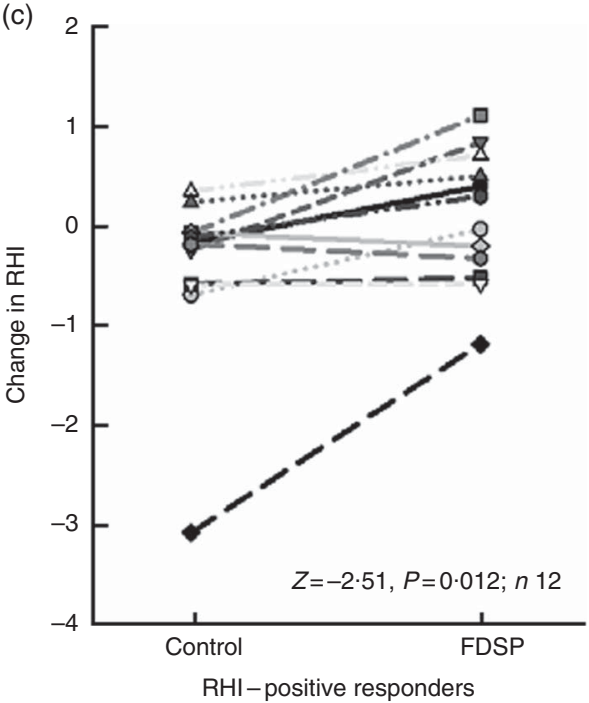

(b)

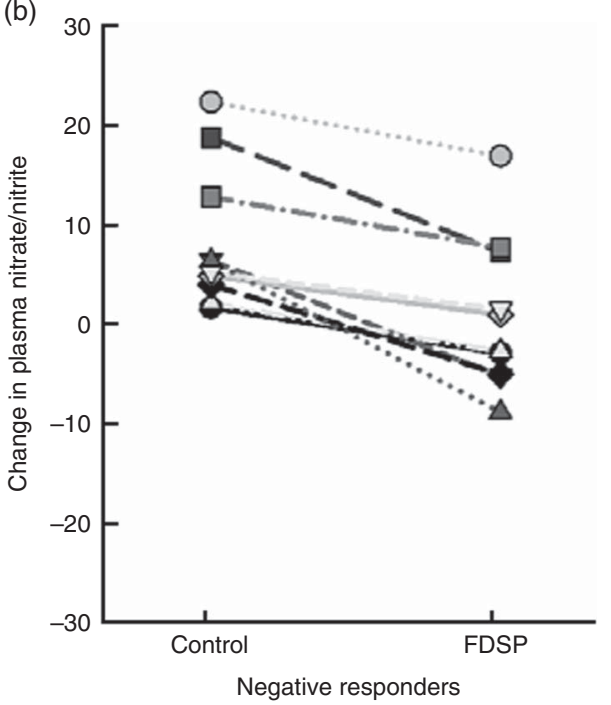

(d)

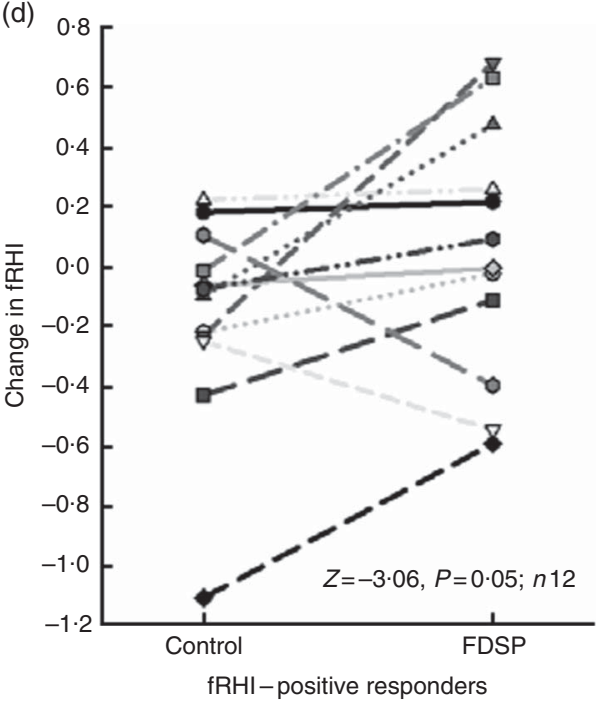

Fig. 3. The differential effects of 1 week of freeze-dried strawberry powder (FDSP) intake on fasting plasma nitrate/nitrite levels and microvascular response in adolescent males (14-16 years of age). (a) Individuals who exhibited a significant positive response in fasting plasma nitrate/nitrite levels after 1 week of FDSP compared with intake of the control powder (5.44 (SD 6.54) v. -3.62 (SD 7.31) $\mu \mathrm{mol} / \mathrm{l}$, respectively, $n$ 12); $v$. (b) who displayed a negative response in plasma nitrate/ nitrite after FDSP intake (FDSP: 0.76 (sD 7.72) $\mu \mathrm{mol} / / \mathrm{v}$. Control: 8.23 (sD 7.11) $\mu \mathrm{mol} / \mathrm{l}$, respectively, $n$ 10). An increase in microvascular function was observed in individuals that who positive plasma nitrate/nitrite responders for both (c) reactive hyperaemia index (RHI) (FDSP: 0.07 (SD 0.67 ) v. Control: -0.45 (SD 0.89 ), respectively; $Z=-2.51, P=0.012, n 12$ ) and (d) Framingham reactive hyperaemia index (fRHI) (FDSP: 0.06 (SD 0.42 ) v. Control: -0.17 (sD 0.35 ), respectively; $Z=-1.96, P=0.050, n 12)$. Statistical testing performed using Wilcoxon's signed-rank test. Each line represents the individual 1-week change in plasma nitrate/nitrite between control and FDSP.

control (non-responders; Fig. 3(a) and (b)), no changes were noted for the non-responders in RHI or fRHI after 1 week of FDSP intake compared with the control group (RHI: $Z=-1.07$, $P=0.285$; fRHI: $Z=-0.66, P=0.508)$. In contrast, the responders showed a significant increase after 1 week of FDSP intake for both RHI and fRHI compared with control powder intake (RHI: $Z=-2.51, P=0.012$; fRHI: $Z=-1.96, P=0.050$; Fig. 3(c) and (d)). No other significant effects were observed between nitrate/nitrite responders and non-responders regarding changes in blood pressure, plasma lipids, fasting blood glucose levels, platelet activation markers, baseline PWA and the peak RHI response measured during the first $2.5 \mathrm{~min}$ of reactive hyperaemia (data not shown).

\section{Discussion}

An increase in endothelial dysfunction has been characterised in paediatric ( $<18$ years of age) populations, which is a troubling observation, as endothelial dysfunction at early ages has been associated with increased $\mathrm{CV}$ risk later in life ${ }^{(9,24-27)}$. A limited number of studies have examined the effects of lifestyle changes on vascular function in obese and overweight children and adolescents. Weight loss has been observed to improve microvascular function ${ }^{(28)}$, and the addition of certain nutrients to the diet, such as folic $\operatorname{acid}^{(29)}$, antioxidant vitamins $^{(30)}$ and certain fatty acids ${ }^{(31,32)}$, may also be of benefit. A limited number of dietary interventions assessing the impact 
of whole foods have also suggested improved vascular function, and our study adds to this literature by demonstrating improvements in vascular function after strawberry powder intake in adolescents who were on average overweight or obese.

In the present study, we observed a significant positive change in RHI in individuals with increased fasting plasma nitrate/nitrite levels after 1 week of FDSP intake. A recent metaanalysis noted that supplementation with inorganic nitrate was associated with a significant improvement in endothelial function, measured by flow-mediated dilation, and that these effects were dose dependent ${ }^{(33)}$. Inorganic nitrate is present in many vegetables and fruits, and it is thought to be a key constituent providing health benefits associated with these foods ${ }^{(34,35)}$. Strawberries contain high levels of nitrate when compared with other types of fruits ${ }^{(36,37)}$, and this may be responsible, in part, for the favourable changes noted in vascular function in our study. Dietary nitrate may serve as a potential source of the potent vasodilator NO. Dietary nitrate is actively reduced to nitrite by bacteria in the oral cavity, with increasing enterosalivary nitrate/nitrite levels associated with increased plasma levels of nitrite, which under specific conditions can be further reduced to bioactive $\mathrm{NO}^{(38)}$. The reduction of nitrate to nitrite and subsequently $\mathrm{NO}$ is potentially $\mathrm{O}_{2}$ independent ${ }^{(39)}$, which is of particular importance in situations of reduced blood flow or increased tissue $\mathrm{O}_{2}$ demand, occurring under conditions such as tissue ischaemia and exercise. Therefore, the intake of nitrate-rich foods and beverages has the potential to improve vascular function in individuals at increased $\mathrm{CV}$ risk. Illustrative of this, the consumption of certain nitrate-rich foods, such as beetroot juice and leafy greens, has been reported to have beneficial effects on blood pressure ${ }^{(40-43)}$

In addition to nitrate, polyphenols in strawberries may also contribute to favourable vascular effects. In vitro and animal studies have shown that anthocyanin treatments can activate, as well as up-regulate, function and/or production of endothelial NO synthase ${ }^{(44-46)}$. A human study with an anthocyanin supplement noted improved endothelium-dependent vasodilation, whereas the addition of a NOS inhibitor abolished the effects $^{(47)}$. Although NO plays a crucial part in regulation of the microvasculature, other factors including input from the sympathetic system and mediators such as adenosine and prostacyclin can contribute to vasodilation/constriction ${ }^{(48,49)}$. Therefore, it is possible that elevated plasma nitrate/nitrite levels may not have been sufficient to act alone in altering the vascular function we report.

In the present study, we did not observe significant positive changes in plasma lipids after 1 week of FDSP intake. Other studies have observed positive changes in plasma lipids after 4 or more weeks of intake ${ }^{(14,15,50)}$. These strawberry feeding trials in adult populations have also reported positive effects on blood lipids, including a decrease in TC and LDL-cholesterol $^{(14,15,50)}$. Similarly, a study that fed mixed berries, which included strawberries, to middle-aged adults at risk for CVD reported an increase in HDL after 8 weeks of intake ${ }^{(51)}$.

Platelets play an integral role in the initiation and progression of atherosclerosis ${ }^{(52)}$, and altered platelet function has been reported in obese children and adolescents ${ }^{(53-56)}$. The effects of strawberries and strawberry drinks made with a similar powder to that used in the present study on platelet function in adults have been examined by several investigations ${ }^{(17,51)}$. Consumption of two daily portions of berries that included strawberries (100 g strawberry purée) for 8 weeks inhibited platelet function as measured by a platelet function analyser ${ }^{(51)}$, and 6 weeks of daily intake of a strawberry beverage (approximately $100 \mathrm{~g}$ fresh strawberries) significantly attenuated PAI-1 concentrations after a high-carbohydrate/fat meal challenge ${ }^{(17)}$. A recent study reported that a 30-d intake of $500 \mathrm{~g}$ of fresh strawberries was significantly associated with a reduced number of activated platelets ${ }^{(57)}$. Although extracts of strawberry polyphenols have been shown to reduce platelet aggregation and soluble P-selectin levels, in vitro ${ }^{(58)}$, in the present study, we observed similar improvements in platelet function in both groups, which might be attributed to other factors present in both control and strawberry powders, such as the amount of $\mathrm{K}$, which has also been associated with reduced platelet reactivity ${ }^{(59)}$.

Limitations of the present study include the short intervention period, which may not have been sufficiently long to elicit positive changes in certain outcomes. Although carryover effects may also be of concern, previous studies have shown that a washout period of $6-10 \mathrm{~d}$ is sufficient to return circulating strawberry phenolic levels to baseline ${ }^{(60,61)}$. Although our control beverage was isoenergetic, it differed in fibre and $\mathrm{K}$ contents, which may have influenced the interaction effects. Our study population contained a mix of normal weight, overweight and obese adolescents, and although the normalweight participants were on the heavier end of the spectrum, their inclusion could have reduced the statistical power needed to detect significant changes. Further limitations may include the sampling time of the study. Although we initially based the timing of sampling on reports of flavonoid- and anthocyanininduced changes in vascular function as soon as 1 week and $1 \mathrm{~h}$ after intake, recent reports have suggested a biphasic response that may be influenced by the presence of colonic metabolites ${ }^{(62)}$, whether or not a similar response would occur with FDSP intake would be of interest in future trials. Finally, a more rigorous measure of compliance might have been helpful, as most of the teenagers in the present study did not return their used packets for verification, even though they verbally reported consumption. Whether the differential in nitrate and vascular responses can serve as a potential form of compliance is of interest, and will need to be confirmed in subsequent investigations. With the above limitations noted, to our knowledge, the present study is the first to examine the effects of consumption of a strawberry powder-based drink on CV outcomes in overweight adolescents, and supports the concept that strawberries can provide vascular health benefits in heavier adolescent males.

\section{Acknowledgements}

The authors thank Matthew Vanness and Grace Lau for their assistance with facilitating study visits, as well as their help with data entry and dietary analysis.

Research funding was provided in part by the California Strawberry Commission (CSC). The CSC had no role in the 
design, analysis or writing of this manuscript. Partial support was also provided by USDA NIFA National Needs Graduate Fellowship to D. D., and by the Agriculture and Food Research Initiative Competitive grant no. 2012-01370 to R. R. H. The UC Davis Comprehensive Cancer Center Optical Biology Core Laboratory is funded by the UC Davis Comprehensive Cancer Center Support Grant awarded by the National Cancer Institute (NCI P30CA093373).

This manuscript is the result of a team research effort emanating from a study designed by C. L. K., R. M. H. and R. R. H. The study was subsequently conducted by D. D. and Ren, with Shindel serving as study physician. The data were analysed by D. D. and R. R. H., with guidance from R. M. H. and C. L. K., who also contributed to the final interpretation. All authors participated in the writing and critical review of the final manuscript, and have read and approved the final version.

The authors declare that there are no conflicts of interest.

\section{References}

1. Ogden CL, Carroll MD, Kit BK, et al. (2014) Prevalence of childhood and adult obesity in the United States, 2011-2012. JAMA 311, 806-814.

2. World Health Organization (2014) Global Status Report on Noncommunicable Diseases 2014. Geneva: WHO.

3. Wang $Z$ \& Nakayama $T$ (2010) Inflammation, a link between obesity and cardiovascular disease. Mediat Inflamm 2010, 535918.

4. Iantorno M, Campia U, Di Daniele N, et al. (2014) Obesity, inflammation and endothelial dysfunction. I Biol Regul Homeost Agents 28, 169-176.

5. Kampoli AM, Tousoulis D, Antoniades C, et al. (2009) Biomarkers of premature atherosclerosis. Trends Mol Med 15, 323-332.

6. Rizvi AA (2009) Cytokine biomarkers, endothelial inflammation, and atherosclerosis in the metabolic syndrome: emerging concepts. Am J Med Sci 338, 310-318.

7. Singhal A (2009) The early origins of atherosclerosis. Adv Exp Med Biol 646, 51-58.

8. Logue J \& Sattar N (2011) Childhood obesity: a ticking time bomb for cardiovascular disease? Clin Pharmacol Ther 90 , 174-178.

9. Pareyn A, Allegaert K, Verhamme P, et al. (2014) Impaired endothelial function in adolescents with overweight or obesity measured by peripheral artery tonometry. Pediatr Diabetes 16, $98-103$.

10. Skinner AC, Steiner MJ, Henderson FW, et al. (2010) Multiple markers of inflammation and weight status: crosssectional analyses throughout childhood. Pediatrics 125 , e801-e809.

11. Defago MD, Elorriaga N, Irazola VE, et al. (2014) Influence of food patterns on endothelial biomarkers: a systematic review. J Clin Hypertens (Greenwich) 16, 1907-1913.

12. Mink PJ, Scrafford CG, Barraj LM, et al. (2007) Flavonoid intake and cardiovascular disease mortality: a prospective study in postmenopausal women. Am J Clin Nutr 85, 895-909.

13. Holt RR, Heiss C, Kelm M, et al. (2012) The potential of flavanol and procyanidin intake to influence age-related vascular disease. J Nutr Gerontol Geriatr 31, 290-323.

14. Basu A, Fu DX, Wilkinson M, et al. (2010) Strawberries decrease atherosclerotic markers in subjects with metabolic syndrome. Nutr Res 30, 462-469.
15. Basu A, Wilkinson M, Penugonda K, et al. (2009) Freeze-dried strawberry powder improves lipid profile and lipid peroxidation in women with metabolic syndrome: baseline and post intervention effects. Nutr J $\mathbf{8}, 43$.

16. Edirisinghe I, Banaszewski K, Cappozzo J, et al. (2011) Strawberry anthocyanin and its association with postprandial inflammation and insulin. Br J Nutr 106, 913-922.

17. Ellis CL, Edirisinghe I, Kappagoda T, et al. (2011) Attenuation of meal-induced inflammatory and thrombotic responses in overweight men and women after 6-week daily strawberry (Fragaria) intake. A randomized placebo-controlled trial. $J$ Atheroscler Thromb 18, 318-327.

18. Heiss C, Jahn S, Taylor M, et al. (2010) Improvement of endothelial function with dietary flavanols is associated with mobilization of circulating angiogenic cells in patients with coronary artery disease. J Am Coll Cardiol 56, 218-224.

19. Balzer J, Rassaf T, Heiss C, et al. (2008) Sustained benefits in vascular function through flavanol-containing cocoa in medicated diabetic patients a double-masked, randomized, controlled trial. J Am Coll Cardiol 51, 2141-2149.

20. Mullen W, Edwards CA, Serafini M, et al. (2008) Bioavailability of pelargonidin-3-O-glucoside and its metabolites in humans following the ingestion of strawberries with and without cream. J Agric Food Chem 56, 713-719.

21. Holt R, Yim S, Shearer G, et al. (2015) Effects of short-term walnut consumption on human microvascular function and its relationship to plasma epoxide content. J Nutr Biochem $\mathbf{6}$, 1428-1466.

22. Hamburg NM, Keyes MJ, Larson MG, et al. (2008) Cross-sectional relations of digital vascular function to cardiovascular risk factors in the Framingham Heart Study. Circulation 117, 2467-2474.

23. Pearson DA, Paglieroni TG, Rein D, et al. (2002) The effects of flavanol-rich cocoa and aspirin on ex vivo platelet function. Thromb Res 106, 191-197.

24. Aatola H, Hutri-Kahonen N, Juonala M, et al. (2014) Prospective relationship of change in ideal cardiovascular health status and arterial stiffness: the Cardiovascular Risk in Young Finns Study. J Am Heart Assoc 3, e000532.

25. Mahmud FH, Hill DJ, Cuerden MS, et al. (2009) Impaired vascular function in obese adolescents with insulin resistance. J Pediat 155, 678-682.

26. Aggoun Y, Farpour-Lambert NJ, Marchand LM, et al. (2008) Impaired endothelial and smooth muscle functions and arterial stiffness appear before puberty in obese children and are associated with elevated ambulatory blood pressure. Eur Heart J 29, 792-799.

27. Bruyndonckx L, Hoymans VY, Frederix G, et al. (2014) Endothelial progenitor cells and endothelial microparticles are independent predictors of endothelial function. J Pediat 165 , 300-305.

28. Bruyndonckx L, Hoymans VY, De Guchtenaere A, et al. (2015) Diet, exercise, and endothelial function in obese adolescents. Pediatrics 135, e653-e661.

29. Pena AS, Wiltshire E, Gent R, et al. (2004) Folic acid improves endothelial function in children and adolescents with type 1 diabetes. J Pediat 144, 500-504.

30. Engler MM, Engler MB, Malloy MJ, et al. (2003) Antioxidant vitamins $\mathrm{C}$ and $\mathrm{E}$ improve endothelial function in children with hyperlipidemia: Endothelial Assessment of Risk from Lipids in Youth (EARLY) Trial. Circulation 108, 1059-1063.

31. Engler MM, Engler MB, Malloy M, et al. (2004) Docosahexaenoic acid restores endothelial function in children with hyperlipidemia: results from the EARLY study. Int $\mathrm{J}$ Clin Pharmacol Ther 42, 672-679. 
32. Dangardt F, Osika W, Chen Y, et al. (2010) Omega-3 fatty acid supplementation improves vascular function and reduces inflammation in obese adolescents. Atherosclerosis $\mathbf{2 1 2}$ $580-585$.

34. Hord NG, Tang Y \& Bryan NS (2009) Food sources of nitrates and nitrites: the physiologic context for potential health benefits. Am J Clin Nutr 90, 1-10.

35. Bondonno CP, Croft KD \& Hodgson JM (2015) Dietary nitrate, nitric oxide and cardiovascular health. Crit Rev Food Sci Nutr 15, 47.

36. Susin J, Kmecl V \& Gregorcic A (2006) A survey of nitrate and nitrite content of fruit and vegetables grown in Slovenia during 1996-2002. Food Addit Contam 23, 385-390.

37. Nabrzyski M \& Gajewska R (1994) [The content of nitrates and nitrites in fruits, vegetables and other foodstuffs]. Rocz Panstw Zakl Hig 45, 167-180.

38. Lundberg JO, Weitzberg E \& Gladwin MT (2008) The nitratenitrite-nitric oxide pathway in physiology and therapeutics. Nat Rev Drug Discov 7, 156-167.

39. Cosby K, Partovi KS, Crawford JH, et al. (2003) Nitrite reduction to nitric oxide by deoxyhemoglobin vasodilates the human circulation. Nat Med $\mathbf{9}, 1498-1505$.

40. Hobbs DA, George TW \& Lovegrove JA (2013) The effects of dietary nitrate on blood pressure and endothelial function: a review of human intervention studies. Nutr Res Rev 26, 210-222.

41. Vanhatalo A, Bailey SJ, Blackwell JR, et al. (2010) Acute and chronic effects of dietary nitrate supplementation on blood pressure and the physiological responses to moderateintensity and incremental exercise. Am J Physiol Regul, Integr Comp Physiol 299, R1121-R1131.

42. Webb AJ, Patel N, Loukogeorgakis S, et al. (2008) Acute blood pressure lowering, vasoprotective, and antiplatelet properties of dietary nitrate via bioconversion to nitrite. Hypertension $\mathbf{5 1}$, 784-790.

43. Wylie LJ, Kelly J, Bailey SJ, et al. (2013) Beetroot juice and exercise: pharmacodynamic and dose-response relationships. J Appl Physiol 115, 325-336.

44. Xu JW, Ikeda K \& Yamori Y (2004) Upregulation of endothelial nitric oxide synthase by cyanidin-3-glucoside, a typical anthocyanin pigment. Hypertension 44, 217-222.

45. Lazze MC, Pizzala R, Perucca P, et al. (2006) Anthocyanidins decrease endothelin-1 production and increase endothelial nitric oxide synthase in human endothelial cells. Mol Nutr Food Res 50, 44-51.

46. Wang Y, Zhang Y, Wang X, et al. (2012) Supplementation with cyanidin-3-O-beta-glucoside protects against hypercholesterolemia-mediated endothelial dysfunction and attenuates atherosclerosis in apolipoprotein E-deficient mice. J Nutr 142, 1033-1037.

47. Zhu Y, Xia M, Yang Y, et al. (2011) Purified anthocyanin supplementation improves endothelial function via NO-cGMP activation in hypercholesterolemic individuals. Clin Chem 57, 1524-1533.
48. Nohria A, Gerhard-Herman M, Creager MA, et al. (2006) Role of nitric oxide in the regulation of digital pulse volume amplitude in humans. J Appl Physiol 101, 545-548.

49. Hedetoft M \& Olsen NV (2014) Evaluation of endothelial function by peripheral arterial tonometry and relation with the nitric oxide pathway. Nitric Oxide 42C, 1-8.

50. Zunino SJ, Parelman MA, Freytag TL, et al. (2012) Effects of dietary strawberry powder on blood lipids and inflammatory markers in obese human subjects. Br J Nutr 108, 900-909.

51. Erlund I, Koli R, Alfthan G, et al. (2008) Favorable effects of berry consumption on platelet function, blood pressure, and HDL cholesterol. Am J Clin Nutr 87, 323-331.

52. Massberg S, Brand K, Gruner S, et al. (2002) A critical role of platelet adhesion in the initiation of atherosclerotic lesion formation. J Exper Med 196, 887-896.

53. Gallistl S (2000) Correlation between cholesterol, soluble P-selectin, and D-dimer in obese children and adolescents. Blood Coagul Fibrinolysis 11, 755-760.

54. Giordano P, Del Vecchio GC, Cecinati V, et al. (2011) Metabolic, inflammatory, endothelial and haemostatic markers in a group of Italian obese children and adolescents. Eur J Pediatr 170, 845-850.

55. Lohse J (2010) Platelet function in obese children and adolescents. Hamostaseologie 30, S126-S131.

56. Desideri G, De Simone M, Iughetti L, et al. (2005) Early activation of vascular endothelial cells and platelets in obese children. J Clin Endocrinol Metab 90, 3145-3152.

57. Alvarez-Suarez JM, Giampieri F, Tulipani S, et al. (2014) One-month strawberry-rich anthocyanin supplementation ameliorates cardiovascular risk, oxidative stress markers and platelet activation in humans. J Nutr Biochem 25, 289-294.

58. Alarcon M, Fuentes E, Olate N, et al. (2015) Strawberry extract presents antiplatelet activity by inhibition of inflammatory mediator of atherosclerosis (sP-selectin, sCD40L, RANTES, and IL-1beta) and thrombus formation. Platelets 26, 224-229.

59. Kimura M, Lu X, Skurnick J, et al. (2004) Potassium chloride supplementation diminishes platelet reactivity in humans. Hypertension 44, 969-973.

60. Bialasiewicz P, Prymont-Przyminska A, Zwolinska A, et al. (2014) Addition of strawberries to the usual diet decreases resting chemiluminescence of fasting blood in healthy subjects-possible health-promoting effect of these fruits consumption. J Am Coll Nutr 33, 274-287.

61. Prymont-Przyminska A, Zwolinska A, Sarniak A, et al. (2014) Consumption of strawberries on a daily basis increases the non-urate 2,2-diphenyl-1-picryl-hydrazyl (DPPH) radical scavenging activity of fasting plasma in healthy subjects. J Clin Biochem Nutr 55, 48-55.

62. Rodriguez-Mateos A, Rendeiro C, Bergillos-Meca T, et al. (2013) Intake and time dependence of blueberry flavonoidinduced improvements in vascular function: a randomized, controlled, double-blind, crossover intervention study with mechanistic insights into biological activity. Am J Clin Nutr 98, 1179-1191. 